\title{
AOIR
}

Selected Papers of \#AoIR2020: The $21^{\text {st }}$ Annual Conference of the Association of Internet Researchers Virtual Event / 27-31 October 2020

\section{"TASTE THIS VIDEO!": FACEBOOK VIDEOS AS EMBODIED EXPERIENCES}

\author{
Hadas Schlussel \\ The Hebrew University of Jerusalem \\ Paul Frosh \\ The Hebrew University of Jerusalem

\section{Introduction}

"Mesmerizing", "compelling" "daydream", "escapism": these epithets, employed by various journalists (Evans, 2016; Greenberg, 2016) were used to describe seemingly banal everyday recipe videos on Facebook. Recipe videos are short clips which show anonymous hands creating desserts, roast chicken, salads, and other culinary delights in less than 50 seconds. The journalists mentioned above were not impressed by the creativity of the recipes themselves, they could not even recall which recipes they had seen. Yet, they clearly remembered the kind of experience they had while watching, and the explicit compulsion they felt to watch more videos. Indeed, the compulsive character of the response they describe may have made recipe videos among Facebook's most popular genres. In February 2020, Tasty - according to Socialbakers, the most popular Facebook community in the world - had garnered more than 100 million followers.

Furthermore, Tasty's distinctive style had been imitated in other recipe videos Facebook Pages, in other genres widespread in social media, such as DIY videos, and in television commercials. This paper suggests the "mesmerizing" power of recipe videos is achieved through the way they use the social media interface to overtly address users' senses, rather than serve informational purposes, and create tactile experiences that embody users' presence in the virtual space.

Trying to understand the significance of recipe videos' popularity as a phenomenon, we analyze recipe videos through a novel three-dimensional model that integrates their semiotic characteristics (visual, auditory, and textual), their interactive and haptic qualities, and their invitation to perceptual engagement and sensorimotor response. All these dimensions, we argue, are crucial to understanding the intersections between video and the context of engagement on social media interfaces. We conclude that Facebook recipe videos are exemplary of a broader category of social media videos

Suggested Citation (APA): Schlussel, H., Frosh, P. (2020, October). "Taste This Video!": Facebook Videos as Embodied Experiences. Paper presented at AolR 2020: The 21 th Annual Conference of the Association of Internet Researchers. Virtual Event: AolR. Retrieved from http://spir.aoir.org. 
which we call hyper-sensory videos: these rise to the challenge of an interruptive context of viewing by presenting heightened multisensory experiences that take precedent over informational use or narrative involvement.

\section{Theoretical background}

Despite their surprising popularity, there is no research literature on Facebook recipe videos and almost no literature on Facebook videos in general. Eder (2018) argues that online videos constitute a medium in their own right, with new participatory and interactive characteristics. Facebook videos possess distinctive attributes adapted to the specific characteristics of their display environment: namely, the Facebook interface's news feed and the mute auto-play feature. The Facebook feed, a continually flowing "social media data-stream" (Hochman, 2014), is ruled by the "attention economy" (Goldhaber, 1997), whereby human attention becomes an increasingly scarce and valuable resource. Therefore, recipe videos compete over users' attention by creating high stimuli, and aesthetically catchy frames that the human eye can perceive easily.

In a sense, this research continues other works that contemplate the perception of media through human sensoria, in case studies like films, fine literature, or artworks (Esrock, 2019; Merleau-Ponty, 1982; Sobchak, 2014). Creating a desire for an intensified presence (Gumbrecht, 2004) and economic power, by shaping human capacities to sense space and time (Ash, 2019), digital media play an important role in understanding our relationships with culture and technology. The overt enhancement of multisensory and sensorimotor experience presented (with such broad popularity) by Tasty videos can shed light on these relationships, potentially reflecting a yearning for embodied presence in increasingly digitized and 'virtual' conditions of social existence.

\section{Methodology}

The corpus was comprised of the most shared recipe videos on Tasty's Facebook page. Tasty was chosen because of its exceptional number of followers and because, watching the videos, we could discern a fixed-style formula, consistent across all Tasty's videos, and imitated by other recipe video creators. The indication of this formula also allowed a relatively small corpus, of 15 videos, which were later transcribed shot by shot. Sharing was chosen as an indicator, not only of active engagement with the video but also of its virality (Wittel, 2011). Videos were chosen according to data extracted using the Netvizz Application (Rieder, 2013).

Using a phenomenological approach, drawing on Seteur's (1992) analysis of telepresence dimensions and Frosh's (2018) analysis of interface aesthetics, we developed a novel three-dimensional analytical framework attuned to the conditions of display in the Facebook newsfeed. This framework addressed 1) the videos' visual, auditory and textual semiotic attributes, 2) the involvement and activation structures of the videos as elements of the Facebook interface, and 3) the embodied, sensory and sensorimotor relations between users and screen devices. 


\section{Analysis}

The analysis foregrounds several dominant tactics recipe videos apply to enhance sensory-motor experience and compete in Facebook's attention economy. These include the remediated amplification of some audio-visual elements and the reduction of others: due to the mute auto-play, the sound was reduced to music only, and subtitles describing the recipe were added. The videos also blurred the line between touch and vision in ways that shift depictive and interface dimensions. All videos are filmed from a high angle in a manner that depicts anonymous hands preparing food, approximating the user's point of view, as well as producing visual continuity between the depicted hands and the hands of the user, holding the mobile screen. At the same time, this representational intersection between vision and touch interrupts the routinized handeye relations of the Facebook interface, making us watch it by stopping the scrollthrough movement of our fingers on the screen. The recipe video thus appeals to the eyes as a touching organ, or even as if the eyes can taste, as it depicts textures and movement up close, in order to re-channel and effectively suspend - albeit temporarily - users' habitual gestural engagements with the mobile interface. Overall, the analysis suggests a new kind of digital format which uses remediation, haptic vision, and gestural interactivity, to adapt video to the embodied attention-structures of social media interfaces.

\section{References}

Ash, J. (2015). The interface envelope: Gaming, technology, power. Bloomsbury Publishing USA.

Eder, J. (2018). Collateral emotions: Political web videos and divergent audience responses. In Cognitive theory and documentary film (pp. 183-203). Palgrave Macmillan, Cham.

Esrock, E. J. (2019). 11 Body Forth in Narrative. Narrative Complexity: Cognition, Embodiment, Evolution, 270.

Frosh, P. (2018). The mouse, the screen and the Holocaust witness: Interface aesthetics and moral response. New Media \& Society, 20(1), 351-368.

Goldhaber, M. H. (1997). The attention economy and the net. First Monday,2(4).

Gumbrecht, H. U. (2004). Production of presence: What meaning cannot convey. Stanford University Press.

Hochman, H. (2014). The social media image. Big Data \& Society, 15(1).

Merleau-Ponty, M. (1982). Phenomenology of perception. Routledge.

Rieder, B. (2013) Studying Facebook via data extraction: the Netvizz application. In: WebSci'13, Paris, France May 2-4 2013, pp. 346-355. New York: ACM. 
Sobchack, V. (2004). Carnal Thoughts: Embodiment and moving image culture (pp. 3553). Univ of California Press.

Steuer, J. (1992). Defining virtual reality: Dimensions determining telepresence. Journal of Communication, 42(4), 73-93.

Wittel, A. (2011). Qualities of sharing and their transformations in the digital age.

International Review of Information Ethics, 15(9), 3-8.

Evans, D. (2016, March 23). Why These Recipe Videos Are Taking Over Your Facebook Wall. The Cut. Retrieved on 24 of September from:

https://www.thecut.com/2016/03/zen-and-the-art-of-the-buzzfeed-tasty-video.html

Greenberg, J. (2016, May 30). Food Has Eaten the Internet and It Tastes Like a Vampire Taco. Wired. Retrieved on 24 of September from:

https://www.wired.com/2016/05/internet-reached-perfection-looks-like-vampire-taco/ 